\title{
Stratégie de validation de méthodes de dosage en bioanalyse en vue d'études pharmacocinétiques et toxicologiques
}

\section{A strategy for validation of bioanalytical methods to support pharmacokinetic and toxicological studies}

Olivier NICOLAS ${ }^{(1)}$, Christine FARENC ${ }^{(2)}$, Françoise BRESSOLLE ${ }^{(1) *}$

(1) Laboratoire de Pharmacocinétique Clinique (2) Laboratoire de Chimie Analytique, Faculté de Pharmacie, Université Montpellier I - FRANCE

*Auteur à qui adresser la correspondance : Dr Françoise BRESSOLLE, Laboratoire de Pharmacocinétique Clinique, Faculté de Pharmacie, Université Montpellier I - BP 14491 - 34093 Montpellier Cedex 5 - FRANCE Tél : 33467548075 - Fax : 33467548075 -E-mail : FBressolle@aol.com

(Reçu le 26 décembre 2003 ; accepté le 15 juin 2004)

\section{$R \mathbf{E} S U M E$}

Lors des études de pharmacocinétiques, toxicocinétiques et lors du suivi thérapeutique des patients, il est important d'utiliser une méthode analytique correctement validée pour obtenir des résultats fiables qui pourront être ainsi interprétés de façon satisfaisante. Dans ce manuscrit les différentes étapes de la validation sont développées; leurs buts étant de démontrer la fiabilité des résultats à la fois pour les principes actifs et les métabolites. A cet effet, les problèmes liés à la qualification de l'équipement analytique et au pré-traitement de l'échantillon (effet matrice) seront abordés. Les critères de validation comprennent la spécificité, la fidélité, l'exactitude, la linéarité, les seuils de quantification et de détection, la robustesse mais aussi tous les essais de stabilité.

\section{MOTS-CLÉS}

Validation analytique, Bioanalyse, Fidélité, Exactitude, Spécificité, Robustesse, Essais de stabilité.

\section{SUMMARY}

Validations of analytical methods are important for the generation of data for pharmacokinetics, toxicokinetics and during therapeutic drug monitoring. It is essential to use well defined and fully validated analytical methods to obtain reliable results that can be satisfactorily interpreted. This manuscript is intended to provide guiding principles for the evaluation of a method's overall performance. For this purpose, problems due to instrument qualification and to sample pretreatment (matrix effect) are considered. The criteria considered are as follows : selectivity, accuracy, precision, linearity, recovery, limits of quantitation and of detection, ruggedness and stability essays.

\section{KEY-WORDS}

Method validation, Bioanalysis, Accuracy, Precision, Specificity, Ruggedness, Stability assays. 


\section{Introduction}

Les méthodes analytiques sont indispensables pour quantifier les médicaments et leurs métabolites dans les prélèvements biologiques. Pour pouvoir être utilisées, ces méthodes doivent être validées. L'objectif de la validation est de produire des résultats fiables et reproductibles. En effet, les données fournies sont cruciales lors de la réalisation d'études de pharmacocinétique, de bioéquivalence, de biodisponibilité ou encore au cours des études toxicologiques chez l'animal puis chez l'homme. La validation des méthodes en bioanalyse est une étape clé du développement de nouvelles molécules jusqu'à l'enregistrement auprès des autorités.

La maîtrise de la qualité des analyses est une préoccupation importante. La démarche à suivre ne se limite pas à la production de procédures dont le suivi assure la traçabilité totale des résultats, conditions nécessaires à l'accréditation. Elle intègre également la qualification de l'appareillage utilisé, son suivi et surtout la validation de la méthode de dosage qui permet de prouver que cette méthode répond bien aux besoins pour lesquels elle a été développée, avec la possibilité de la transférer dans un autre laboratoire. Ceci impose une caractérisation précise des critères de la méthode : sélectivité, spécificité, exactitude, répétabilité, reproductibilité, linéarité, seuils de quantification et de détection, et robustesse (1-6).

Les systèmes analytiques sont aujourd'hui capables de générer une très grande quantité de données. Cette profusion d'informations parfois redondantes peut nuire à l'exploitation et à l'interprétation des données. La mise en ouvre d'outils mathématiques et statistiques avancés se révèle alors indispensable afin d'extraire l'information la plus pertinente.

Le but de cet article est de présenter les différents critères de validation d'une méthode de dosage en bioanalyse, en vue de son application à l'analyse d'échantillon en routine (études pharmacocinétiques et toxicocinétiques, études toxicologiques, suivi thérapeutique). Nous traiterons essentiellement la validation des méthodes chromatographiques qui sont les plus utilisées puis nous dirons quelques mots sur la spécificité de la validation des méthodes immunologiques. Les principales difficultés rencontrées et les solutions envisagées seront discutées.

\section{Qualification de l'équipe- ment analytique}

Dans le cadre de l'assurance qualité et des bonnes pratiques de laboratoire, avant toute validation de métho- de, il est nécessaire de qualifier l'équipement analytique du laboratoire et de vérifier régulièrement l'aptitude des appareils (7). Ceci permet de garantir que le système est correctement étalonné et testé. Un document concernant la validation de l'équipement par le fournisseur, la fiche de vie et les opérations de maintenance doit être disponible pour chaque appareil.

Le processus de validation commence chez le fournisseur. A cette étape, l'équipement analytique et le logiciel sont développés et validés selon les normes ISO/IEC 17025 (8) et la norme 21 CFR part 11 de la FDA (9) relative aux systèmes informatiques.

Lors de la mise en service d'un appareillage, il faudra effectuer la qualification à l'installation, la qualification opérationnelle et la qualification des performances. Il s'agit d'une qualification fonctionnelle réalisée sur le site de l'utilisateur. La qualification à l'installation doit permettre de détecter les défauts de livraison et de l'environnement de l'installation. La qualification opérationnelle a pour but de vérifier les fonctions qui seront utilisées lors des analyses. Si celles ci sont conformes, l'utilisateur peut effectuer une série de mesures afin de qualifier les performances, c'est à dire de déterminer si l'appareil répond à ses besoins (7).

Après une périóde d'utilisation, il est nécessaire de mettre en place des procédures de maintenance. Il est également recommandé d'effectuer un test vérifiant l'efficacité du système d'analyse au début et au cours d'une étude. Les différents paramètres d'un système chromatographique à valider sont (6) :

- le facteur de capacité $\left(\mathrm{k}^{\prime}=\left[\mathrm{tr}_{\text {(analyte) }}-\mathrm{tr}_{\text {(non retenu) }}\right] / \mathrm{tr}_{\text {(non retenu) }}\right.$ avec $t r=$ temps de rétention) le pic de l'analyte doit être bien séparé du pic du non retenu, généralement k' doit être $>2$,

- la répétabilité de l'injection (coefficient de variation $<1 \%$; nombre d'injection $\mathrm{n}=5$ ),

- le temps de rétention relatif,

- la résolution (Rs $=2 \times\left(\operatorname{tr}_{2}-\operatorname{tr}_{1}\right) /\left(\mathrm{L}_{2}+\mathrm{L}_{1}\right)$ avec $\operatorname{tr}_{1}, \operatorname{tr}_{2}$ temps de rétention des composés 1 et 2 et $\mathrm{L}_{1}, \mathrm{~L}_{2}$ largeur du pic à la base des composés 1 et 2) a) entre les pics d'intérêt (par exemple analyte et étalon interne ; molécule mère et métabolites ; énantiomères) et b) avec les composés interférents de la matrice. Généralement Rs doit être $>2$,

- la symétrie des pics $\left(\mathrm{As}=\mathrm{L}_{5 \%} /(2 \mathrm{xF})\right.$ avec $\mathrm{L}_{5 \%}$ largeur du pic mesurée à $5 \%$ de la hauteur et $\mathrm{F}$ distance entre la perpendiculaire abaissée du maximum du pic et le bord d'entrée à $5 \%$ de sa hauteur) qui doit être comprise entre 0.8 et 1,5 ,

- le nombre de plateaux théoriques $\left(\mathrm{N}=16(\mathrm{tr} / \mathrm{L})^{2}\right)$ qui doit être calculé pour chaque nouvelle colonne en utilisant l'analyte ou l'étalon interne. 


\section{Choix de la méthode de dosage et élimination des composés interférents de la matrice}

Le choix de la méthode d'analyse et surtout le pré-traitement de l'échantillon biologique sont des étapes clés qui précèdent la validation proprement dite. En effet, les matrices biologiques contiennent une multitude de composés endogènes (protéines dans le plasma, acides gras dans l'urine) souvent en concentrations bien plus fortes que les analytes ou leurs métabolites qui peuvent interférer avec les composés à doser. Le développement d'une méthode de dosage comprend :

- Le choix du solvant dans lequel l'analyte sera solubilisé et sa stabilité. Il faudra vérifier le risque d'interaction contenant/analyte. Il est ainsi possible de sylaniser la verrerie afin d'empêcher l'adsorption de l'analyte sur le verre.

- Le choix de la matrice. La méthode devra être validée dans chaque matrice dans laquelle le composé sera dosé. Il faudra ensuite s'assurer que les prélèvements soient conservés dans de bonnes conditions. En effet, toutes variations de $\mathrm{pH}$ du plasma ou du sang ex vivo peut modifier le pourcentage de liaison aux protéines plasmatiques (10-12) et la stabilité des composés (13). De même, il faudra s'assurer que l'anticoagulant choisi n'a pas d'influence sur les performances de la méthode analytique utilisée pour quantifier le composé $(14,15)$.

- Le choix de l'étalon interne. Généralement, l'étalon interne est un composé de même série chimique que l'analyte. L'étalon interne doit s'extraire dans les mêmes conditions et doit avoir un temps de rétention différent de l'analyte. Il est recommandé d'utiliser des analogues deutérés de l'analyte lorsque le système de détection est la spectrométrie de masse (SM).

- Le choix des conditions de pré-traitement de l'échantillon à partir de la matrice choisie. Il peut s'agir d'extractions solide-liquide, liquide-liquide ou d'une simple précipitation ou démixtion.

- Le choix du système d'analyse (chromatographie liquide haute performance (CLHP) ou chromatographie en phase gazeuse (CG)).

- Le choix du système de détection (SM, UV, fluorescence...).

- Le choix de la relation entre concentration de l'analyte dans la matrice et réponse du détecteur.

- Le temps d'analyse.

Les différentes étapes du développement de la métho- de analytique doivent être écrites et archivées afin de justifier les choix et d'être consultables pendant l'étape de validation.

\section{Effet matrice}

Le but du pré-traitement des échantillons biologiques est l'élimination des composés endogènes de la matrice qui peuvent soit interférer avec les composés à doser soit être à l'origine d'effet matrice. Durant ces dix dernières années, les techniques de chromatographie liquide haute performance couplées à la spectrométrie de masse (CLHP/SM et CLHP/SM/SM.) sont devenues un outil analytique puissant. Ces techniques sont sensibles et spécifiques et permettent l'analyse de traces dans des mélanges complexes ; elles pourraient, grâce à ces caractéristiques, permettre de s'affranchir de l'étape préalable de pré-traitement des échantillons. Mais une des limites de ces méthodes est leurs susceptibilités à l'effet matrice $(16,17)$. L'effet matrice est défini comme l'effet de substances résiduelles de la matrice, co-éluées, sur l'ionisation de l'analyte d'intérêt. La suppression ou la potentialisation de la réponse de l'analyte est accompagnée d'une diminution de la fidélité et de l'exactitude de la méthode. L'effet matrice est dépendant du type d'ionisation, du pré-traitement de l'échantillon et du type de matrice biologique (plasma, urine, salive). Lors d'une étude réalisée sur la morphine (18) les effets matrices sont observés pour tous les types d'ionisation mais la technique APCI (Atmospheric pressure chemical ionization) semble moins sensible à l'effet matrice que la technique électrospray (ESI). Bien que l'échantillon soit plus propre après une étape d'extraction sur phase solide, l'étape de pré-concentration augmente la concentration de l'analyte d'intérêt mais aussi celle des substances interférentes provenant des fluides biologiques. Ainsi l'injection directe ou après une simple dilution peut limiter l'effet matrice. Cependant cette étape de pré-concentration peut être nécessaire pour obtenir une sensibilité satisfaisante. L'effet matrice dépend aussi de la nature du fluide biologique, les composés de la matrice interférent à différents temps d'élution et avec une intensité différente tout au long de l'analyse. Dans l'urine les interférences proviennent de composé hydrophiles et très souvent de sels inorganiques. Dans la salive et le plasma, l'intensité des interférences est plus importante et concerne à la fois des dérivés hydrophiles et hydrophobes. Normalement, l'utilisation comme étalon interne, d'un analogue isotope stable qui sera co-élué avec l'analyte, peut permettre de s'affranchir des répercussions sur la fidélité/exactitude puisque le rapport des réponses analyte/étalon interne sera conservé. Mais 
une étude récente (19) concernant le dosage de l'acide mévalonique dans le plasma et l'urine a montré que dans certaines conditions, l'utilisation d'analogues deutérés comme étalon interne ne suffit pas à s'affranchir de l'effet matrice.

\section{Procédure de validation}

La validation d'une méthode en bioanalyse comprend une succession de procédures afin de démontrer que la méthode utilisée permet de quantifier un analyte dans une matrice particulière pour une application précise. Différents paramètres permettent de définir l'acceptation d'une méthode analytique : l'exactitude, la fidélité, la sélectivité, la spécificité, la répétabilité, la reproductibilité, la robustesse et enfin la stabilité de l'analyte au cours des différentes étapes de pré-traitement et dans la matrice (1-6). La validation est un événement dynamique et adaptable en fonction de son application et des conditions opératoires. Ainsi, la sélectivité de l'analyse vis à vis des composés endogènes de la matrice, des métabolites éventuels, des comédications ou de l'anticoagulant choisi pour collecter l'échantillon biologique doit être évaluée.

Parmi les différents niveaux de validation, on discerne la validation complète, la validation partielle et les validations croisées.

Une validation complète est nécessaire dans les conditions suivantes :

- Dosage d'un analyte pour la première fois.

- Utilisation de la méthode mise au point pour le dosage de la molécule mère au dosage des métabolites.

Les différentes étapes d'une validation complète sont schématisées Figure 1.

Une validation partielle est une modification d'une méthode déjà validée qui ne requiert aucune validation complète (1-3). Les changements qui nécessitent une validation partielle peuvent être : le transfert d'une méthode analytique entre laboratoires, une modification d'appareillage ou de logiciel de gestion, un changement d'espèce pour une matrice donnée ou un changement de matrice dans une même espèce. Les paramètres qui devront faire l'objet de cette validation sont 1) l'étude de la relation concentration / réponse (6 gammes d'étalonnage à des jours différents) et 2) la vérification de l'exactitude et de la fidélité (dosage d'échantillons contrôle-qualité : bas, milieu et haut de gamme à des jours différents $(n=6))$.

Une validation croisée est réalisée si deux méthodes analytiques différentes sont utilisées pour générer des données dans une même étude (1-3). La validation croisée s'effectue par une comparaison statistique des



Figure 1 : Différentes étapes d'une validation complète.

paramètres de validation de chacune des méthodes. L'une d'elles servant de référence et l'autre de test, puis inversement. Si l'étude est conduite de façon multicentrique, la validation croisée s'effectue par le dosage d'échantillons contrôle-qualité de concentrations connues et d'échantillons de plasma de patients dans chacun des laboratoires concernés. Les résultats sont ensuite analysés statistiquement.

Pour les matrices biologiques impossibles à se procurer en quantité suffisante pour une validation (par exemple liquide céphalorachidien, sang de nouveaux-nés, etc.), une matrice de remplacement peut être utilisée (3). Dans ce cas, la validation est réalisée dans la matrice de remplacement puis une validation croisée est réalisée avec la matrice à étudier. La procédure est la suivante : des échantillons contrôle-qualité incluant le seuil de quantification sont préparés dans la matrice de remplacement et dans la matrice à étudier ; ils sont dosés par rapport à une gamme d'étalonnage préparée dans la matrice de remplacement. La méthode est considérée 
validée si la fidélité et l'exactitude sur l'analyse des échantillons contrôle-qualité répondent aux critères cités ci-dessous. De plus, il est important de vérifier la spécificité de la méthode avec la matrice à étudier.

\section{Spécificité / Sélectivité :}

La spécificité est la capacité de quantifier un analyte sans équivoque en présence de composés endogènes de la matrice. La sélectivité est l'aptitude d'une méthode analytique à quantifier l'analyte et à le séparer des produits de dégradation, des métabolites et des composés coadministrés.

L'examen de la sélectivité s'effectue par l'analyse d'un blanc matrice (plasma, urine,...) en démontrant l'absence de réponse au temps de rétention de l'analyte par comparaison avec une solution pure de l'analyte. Il est recommandé de l'évaluer sur au moins 6 sources différentes d'une même matrice. Une autre approche, dans le cas d'une relation linéaire non pondérée, entre concentration de l'analyte et réponse du détecteur, est d'effectuer un test de Student sur l'ordonnée à l'origine qui doit être non statistiquement différent de 0 .

En présence de métabolites et de produits endogènes, se pose le problème de la pureté du pic de l'analyte. Différentes méthodes peuvent être employées pour s'assurer de la pureté du pic et de la spécificité de la méthode :

- l'emploi d'un détecteur à barrette de diode

- l'emploi d'un détecteur plus spécifique type spectromètre de masse

- L'emploi de différentes colonnes chromatographiques de sélectivités différentes dans différentes conditions chromatographiques (chromatographie multidimensionnelle)

- L'étude d'échantillons de patients ayant reçu le médicament, collectés à des temps de prélèvements différents par comparaison au plasma de ces mêmes patients recueilli avant administration

- Le rajout de quantités connues d'analyte à l'échantillon

La sélectivité est un paramètre important notamment à la concentration correspondant au seuil de quantification de la méthode analytique (4).

\section{Gamme d'étalonnage :}

Il est nécessaire d'utiliser un nombre de points de gamme d'étalonnage suffisant pour définir la relation entre la concentration de l'analyte dans la matrice et la réponse correspondante du détecteur. Ces points de gamme sont obtenus en supplémentant la matrice d'une quantité croissante connue de l'analyte en solution pure (solution de travail). Le volume ajouté doit être inférieur ou égal à $2 \%$ du volume total de l'échantillon de façon à conserver l'intégrité de la matrice. En ce qui concerne la quantification de(s) composé(s) dans un échantillon tissulaire, il est nécessaire au préalable d'homogénéiser l'échantillon (ultraturax, congélation dans l'azote liquide puis broyage (21)). Lors de la réalisation de gammes d'étalonnage dans des tissus, après ajout de l'analyte, un temps de contact est nécessaire afin de permettre la liaison de l'analyte aux protéines tissulaires (un bon procédé est un contact de $2 \mathrm{~h}$ à $4^{\circ} \mathrm{C}(21)$ ).

Il est nécessaire d'établir une gamme d'étalonnage pour chaque analyte à quantifier dans la matrice (molécule mère, métabolites, énantiomères...). Cette gamme d'étalonnage doit être composée d'au moins 6 à 8 points (sans le blanc matrice, qui est un échantillon de la matrice biologique exempt d'analyte) ; le point le plus faible de la gamme correspond en général au seuil de quantification. La courbe d'étalonnage décrivant la relation entre la concentration de l'analyte et la réponse doit être décrite par le modèle mathématique le plus simple possible (relation linéaire pondérée ou non pondérée ; quadratique ; log-linéaire....).

Au moins 5 gammes d'étalonnage sont réalisées le même jour, à partir de la même solution mère, pour tester la répétabilité de la méthode (variabilité minimum) et au moins 6 gammes sont réalisées à des jours différents, à partir de solutions mères différentes, pour tester sa reproductibilité (variabilité maximum).

Pour chaque point de gamme réalisé le même jour et à des jours différents, à partir de l'équation reliant réponse et concentration ajoutée $\left(\mathrm{C}_{\mathrm{REF}}\right)$, une concentration est recalculée $\left(\mathrm{C}_{\mathrm{CALC}}\right)$ ainsi que l'erreur relative (ER\% $\left.=\left[\left(\mathrm{C}_{\mathrm{REF}}-\mathrm{C}_{\mathrm{CALC}}\right) / \mathrm{C}_{\mathrm{REF}}\right] \times 100\right)$ correspondante.

Pour chaque concentration ajoutee, une concentration recalculée moyenne est déterminée ainsi que le coefficient de variation correspondant. Ce coefficient doit être inférieur à $15 \%$, mais on tolère $20 \%$ au seuil de quantification (1-5).

Afin de vérifier le bon ajustement de la courbe aux points expérimentaux, dans l'intervalle des concentrations fixées, les tests statistiques suivants doivent être réalisés :

- Etude de la distribution des résidus (concentrations ajoutées-concentrations calculées) qui doit suivre une loi normale et centrée sur zéro.

- Comparaison des concentrations calculées aux concentrations ajoutées par régression linéaire (comparaison de la pente à 1 et de l'ordonnée à l'origine à zéro par un test de Student)

- Calcul du biais et de son intervalle de confiance à $95 \%$.

$$
\text { Biais }=\frac{1}{n} \sum_{i=1}^{i=n}\left[C_{C A L C}-C_{R E F}\right]
$$


Dans le cas de relation linéaire entre réponse et concentration, le test du «lack of fit» doit être vérifié. La pente de la droite d'étalonnage doit être statistiquement différent de zéro, l'ordonnée à l'origine non statistiquement différente de zéro et le coefficient de corrélation non statistiquement différent de 1.

Il est possible d'exclure des points de gamme, cependant un minimum de 5 points (sans le blanc) doit être conservé. Ce choix s'effectue à partir du calcul de l'erreur relative sur les concentrations recalculées $(>15 \%)$.

\section{Coefficient d'extraction :}

Le coefficient d'extraction absolu est mesuré par le pourcentage de l'analyte retrouvé après pré-traitement de la matrice par comparaison à la même concentration de l'analyte en solution pure. Afin d'étudier les effets de la matrice, il est possible de calculer le rendement de l'extraction (ou coefficient d'extraction relatif) par l'utilisation de blancs matrices prétraités, supplémentés à la concentration théorique étudiée.

Le rendement de l'extraction doit être étudié à trois niveaux de concentration (bas, moyen et haut). Chaque concentration est analysée au moins 3 fois. Le rendement doit être indépendant de la concentration étudiée.

Le coefficient d'extraction doit aussi être déterminé pour l'étalon interne. Ce rendement doit être égal à $+/-15 \%$ celui de l'analyte (4).

\section{Fidélité et exactitude :}

La fidélité et l'exactitude mesurent l'erreur de la mesure analytique et sont les premiers critères étudiés pour apprécier la qualité d'une méthode. L'exactitude est une valeur absolue. Elle correspond au pourcentage retrouvé par rapport à la concentration théorique : (concentration recalculée / concentration théorique) x100. L'exactitude doit être comprise entre 85 et 115 $\%$, sauf pour la concentration du seuil de quantification où elle doit être comprise entre 80 et $120 \%$ (1-5). La fidélité décrit la différence des mesures obtenues en appliquant à partir d'un lot homogène de matrice une même procédure. Elle fournit une indication sur les erreurs liées au hasard (6). La fidélité est donnée par le coefficient de variation affecté à la moyenne des concentrations recalculées. Elle ne doit pas excéder 15 $\%$ sauf pour l'échantillon correspondant au seuil de quantification où une valeur de $20 \%$ est admise (1-5).

La fidélité et l'exactitude sont déterminées par le dosage d'échantillons contrôle-qualité de concentrations connues du produit à doser dans la matrice, par rapport à une gamme d'étalonnage, six fois le même jour et au moins six fois à des jours différents. Trois niveaux de concentration sont étudiées : un bas de gamme (1.5 le seuil de quantification), un milieu de gamme et un haut de gamme (3/4 du point de haut de gamme). Ces échantillons contrôle-qualité sont préparés à partir de solutions de travail différentes de celles utilisées pour préparer la gamme d'étalonnage.

Tout échantillon de concentration supérieure au dernier point de gamme, ne peut être extrapolé à partir de la gamme d'étalonnage. Pour des concentrations supérieures au plus haut point de gamme, il est nécessaire de diluer les échantillons biologiques par de la matrice non traitée. Ces essais de dilution doivent au préalable être validés en terme de fidélité et d'exactitude à l'aide du dosage d'échantillons contrôle-qualité, dilués dans les mêmes proportions. Ces échantillons sont dosés au moins 6 fois chacun par rapport à une gamme d'étalonnage. Les concentrations calculées ne doivent pas être différentes de plus de $+/-15 \%$ par rapport aux concentrations théoriques corrigées du facteur de dilution (4).

\section{Seuil de quantification, seuil de détection et sensibilité :}

Le premier point de la gamme d'étalonnage correspond le plus souvent au seuil de quantification (1). A cette concentration, la fidélité doit être de $20 \%$ et l'exactitude doit être comprise entre 80 et $120 \%$. Le dosage d'échantillons contrôle-qualité $(n=6)$ à la concentration du seuil de quantification permet de calculer ces deux paramètres avec précision. La concentration correspondant au seuil de quantification ne doit pas être confondue avec le seuil de détection, qui correspond à la quantité minimale d'analyte que l'on peut observer mais pas quantifier de façon précise. Des concentrations inférieures au seuil de quantification peuvent être notées comme présentes mais ne doivent pas être interprétées.

La sensibilité de la méthode analytique, à ne pas confondre avec le seuil de détection, est déterminée par la pente de la relation réponse/concentration. Une méthode est dite sensible si un faible changement de concentration provoque une grande variation de réponse du détecteur

\section{Robustesse :}

La robustesse est la capacité d'une méthode analytique à rendre des résultats valables en présence de modifications limitées des conditions expérimentales ou environnementales $(22,23)$. Ces changements délibérés reflètent ceux qui peuvent se produire quand la méthode est utilisée par un autre laboratoire, un autre manipulateur, lorsqu'il y a un changement d'appareillage, etc. Une stratégie pour tester la robustesse d'une méthode chromatographique a été récemment publiée par Nijhuis et al. (22). 


\section{Stabilité :}

On distingue deux types de stabilité : la stabilité de l'analyte au cours des différentes étapes du pré-traitement et la stabilité de l'analyte dans l'échantillon biologique.

- Stabilité au cours des différentes étapes du pré-traitement.

La stabilité d'un analyte dépend de ses propriétés physico-chimiques et des conditions de stockage et de conservation.

Les stabilités des solutions mères et de travail des différents analytes doivent être vérifiées dans des conditions normales de laboratoire en terme de température, d'humidité et de lumière pendant au moins $6 \mathrm{~h}$ par rapport à une solution préparée extemporanément. Les conditions de stockage de ces solutions doivent être également clairement définies $\left(4^{\circ} \mathrm{C},-20^{\circ} \mathrm{C}\right.$, etc). Par ailleurs, la stabilité des analytes doit être vérifiée au cours de toutes les étapes du pré-traitement de l'échantillon (stabilité dans le solvant organique, dans les extraits secs, sur le passeur automatique d'échantillons, etc). En solutions pures, l'analyte et l'étalon interne, sont considérés comme stables si l'écart par rapport à la concentration théorique n'excède pas $2 \%$. Une différence jusqu'à $5 \%$ est tolérée pour les essais de stabilité dans les extraits secs et sur le passeur automatique d'échantillons.

\section{- Stabilité dans l'échantillon biologique}

La stabilité de l'analyte dans la matrice est déterminée par le dosage d'échantillons contrôle-qualité à trois niveaux de concentration (bas, moyen et haut), extemporanément, puis après différents temps de conservation à différentes températures (chaque niveau de concentration est dosé au moins 3 fois).

Il est nécessaire de vérifier deux types de stabilité : la stabilité à court terme $\left(+20^{\circ} \mathrm{C},+4^{\circ} \mathrm{C},-20^{\circ} \mathrm{C}\right)$ et la stabilité à long terme $\left(-20^{\circ} \mathrm{C},-80^{\circ} \mathrm{C}\right)$. Ces essais de stabilité seront réalisés dans toutes les matrices dans lesquelles on sera amené à doser l'analyte.

Des essais de stabilité de l'analyte dans le sang total peuvent également être réalisés afin de valider la procédure de prélèvement sanguin.

Des essais de congélation / décongélation ( 3 cycles) doivent être aussi effectués.

Les concentrations obtenues après stockage ne doivent pas varier de plus de $10-15 \%$ par rapport à la concentration théorique.

\section{Application en routine}

Il est nécessaire de vérifier les performances du système chromatographique (facteur de capacité, résolution et symétrie des pics, nombre de plateaux théoriques, etc) avant chaque série d'analyse à l'aide d'une solution test contenant les analytes. Il est également importants de définir les critères de changement de la colonne analytique. Généralement on admet que la colonne analytique doit être changée lorsque le nombre de plateaux théoriques chute de 40 à $50 \%$ par rapport à la valeur initiale de la colonne neuve. Afin de protéger la colonne analytique, une procédure écrite doit être mise en place concernant le remplacement de la précolonne ou du filtre (en CLHP/SM). Une bonne pratique est d'effectuer ce remplacement tous les 150 à 200 échantillons.

La concentration des échantillons inconnus est déterminée par rapport à une gamme d'étalonnage. Le même analyste doit préparer la gamme et les échantillons à doser. Pour chaque série d'analyse, des échantillons contrôle-qualité (bas, milieu et haut de gamme) sont utilisés pour accepter ou non les résultats. Ces échantillons contrôle-qualité sont préparés et stockés $\left(-20^{\circ} \mathrm{C}\right.$ par exemple) en même temps que les échantillons des patients. A chaque série d'analyse, des échantillons contrôle-qualité sont décongelés puis dosés en même temps que les échantillons inconnus. Leur nombre dépend du nombre total d'échantillons inconnus à analyser. En général, 6 échantillons contrôle-qualité sont dosés tous les 20 échantillons inconnus. Ils doivent être distribués aléatoirement sur le passeur automatique d'échantillons au sein des échantillons à doser. Pour que l'analyse soit acceptée, la concentration calculée pour 4 des 6 échantillons contrôle-qualité doit être de $+/-15 \%$ par rapport aux concentrations ajoutées ( $20 \%$ au seuil de quantification), de plus un contrôle - qualité de chaque concentration doit être correct (4).

Au cours d'une étude il peut arriver que le nombre d'échantillons contrôle-qualité congelés soit insuffisant. Une nouvelle série sera alors préparée ; elle devra être validée par rapport aux anciens échantillons.

\section{Méthodes immunologiques}

Malgré l'utilisation très large des techniques chromatographiques, les méthodes immunologiques (méthodes immumunoradiométriques (RIA, IRMA) et immunoenzymatiques (ELISA, EIA)) restent très utilisées pour certaines applications en bioanalyse supportant le développement de nouveaux composés. De nombreux critères utilisés pour la validation des méthodes 
chromatographiques discutés plus haut sont également applicables aux méthodes immunologiques. Cependant, ces dernières possèdent des particularités qui doivent être considérées lors de la validation. Ces particularités ont été discutées dans les recommandations de la «Food and Drug Administration» (FDA) (1). De plus, des recommandations particulières ont été émises pour les études pharmacocinétiques et toxicocinétiques concernant les macromolécules par une sous commission de «l'American Association of Pharmaceutical Scientists» (AAPS), le Ligand Binding Assay Bioanalytical Focus Group (LBABFG) (24)

\section{Sélectivité :}

Comme pour les méthodes chromatographiques, les méthodes immunologiques doivent être spécifiques de l'analyte. Deux types d'interférences peuvent altérer la sélectivité.

\section{1) L'interférence avec des substances ayant des proprié- tés physico-chimiques similaires à celles de l'analyte.}

La sélectivité dépend de la spécificité de l'anticorps ou de la paire d'anticorps pour l'analyte. Elle sera évaluée vis à vis de substances ayant des propriétés physicochimiques similaires à celles de l'analyte. Ainsi, la réponse des métabolites, des produits de dégradation de l'analyte, des médicaments co-administrés, ou de variants endogènes de l'analyte doit être évaluée séparément et en présence de l'analyte d'intérêt.

Quand cela est possible, pour les petites molécules, les techniques immunologiques doivent être comparées avec la méthode de référence (comme par exemple la CLHP couplée à la SM). La sélectivité peut être améliorée par l'addition d'une étape préalable de pré-traitement évitant ainsi les problèmes liés à la variabilité de la matrice.

Les caractéristiques des macromolécules comme les protéines thérapeutiques rendent difficile et parfois impossible l'extraction avant l'analyse. De plus leurs bio-transformations peuvent ou non altérer l'antigénécité de la protéine. Ainsi, une question reste souvent posée, est ce que la technique permet ou non de différencier les formes pharmacologiquement actives et inactives de la protéine. C'est pourquoi les études de spécificité peuvent être complétées par des expériences permettant d'établir la carte des épitopes ou par des techniques de séparation (par exemple, la CLHP, la «Fast Protein Liquid Chromatography, FPLC», l'électrophorèse sur gel) afin de caractériser la nature de l'immunogénécité de l'échantillon biologique. Des discordances dans la mesure des concentrations entre deux méthodes immunologiques résultent le plus souvent de différences entre leur capacité à différencier la protéine intacte et les formes clivées par protéolyse.

\section{2) L'effet matrice}

Des interférences non spécifiques causées par des facteurs non cités plus haut peuvent affecter la réaction antigène-anticorps. Ces interférences non spécifiques sont regroupées sous le terme «d'effet matrice». Ces facteurs incluent l'hyperlipidémie, l'hémolyse, la force ionique, le $\mathrm{pH}$, la viscosité, les protéines plasmatiques (par exemple, les protéines du complément et les facteurs rhumatoïdes), les anticoagulants, les protéases, les auto-anticorps et les anticorps anti-IgG. Pour évaluer ces interférences non spécifiques, il est recommandé lorsque cela est possible de tester au moins 6 lots de matrice biologique. Lorsque, les méthodes immunologiques mesurent l'analyte directement dans la matrice biologique sans pré-traitement de l'échantillon, l'effet matrice est évalué, en comparant les résultats d'une gamme d'étalonnage réalisée dans le milieu biologique à ceux obtenus avec une gamme réalisée dans un tampon. La dilution avec des tampons contenant des surfactants ou des agents chélateurs (Tween-20, Triton X100 , ou EDTA) peut être utile pour minimiser les interférences non-spécifiques (25). Si la dilution n'est pas suffisante une étape de précipitation des protéines ou d'extraction deviendra nécessaire. Dans ce cas, le même protocole de pré-traitement sera réalisé pour les points de la gamme, les échantillons contrôle-qualité et les échantillons à doser. En derniers recours, des prélèvements réalisés avant l'administration d'un xénobiotique à un sujet, peuvent être utilisés pour préparer les points de la gamme d'étalonnage et les échantillons contrôle-qualité qui seront utilisés lors du dosage des échantillons de ce sujet.

\section{Quantification :}

Les caractéristiques des techniques immunologiques rendent le choix du modèle reliant la réponse aux concentrations plus compliqué que pour les techniques chromatographiques. Les gammes d'étalonnage ne sont pas linéaires. Le plus souvent une équation logistique avec 4 ou 5 paramètres est utilisée. De plus, toujours en raison de cette non linéarité, l'erreur n'est pas une fonction constante de la réponse moyenne (hétéroscédasticité). Ainsi le nombre de points d'une gamme d'étalonnage nécessaire pour déterminer la relation mathématique entre réponse et concentration est supérieur à celui nécessaire lors de l'utilisation de méthodes chromatographiques. Pour établir le modèle lors du développement de la méthode (24), il est recommandé de réaliser la gamme d'étalonnage avec un minimum de 10 concentrations chacune dosée deux fois. Des concentrations en dehors de l'intervalle de quantification (point d'encrage ou «Anchoring points»), ayant des réponses proches des deux régions asymptotiques de la courbe sigmoïde, peuvent être 
incluses pour faciliter la modélisation et pour améliorer l'exactitude et la fidélité des échantillons inconnus ayant des concentrations proches des deux limites de quantification (Figure 2). Au cours de la validation, le modèle de régression non linéaire devra être confirmé par la réalisation d'un minimum de 6 gammes indépendantes. Ce modèle peut être confirmé au cours des analyses réalisées pour déterminer la fidélité et l'exactitude. Un modèle est acceptable lorsqu'à chaque concentration de la gamme d'étalonnage, la moyenne des erreurs relatives n'excède pas $10 \%$ (25).

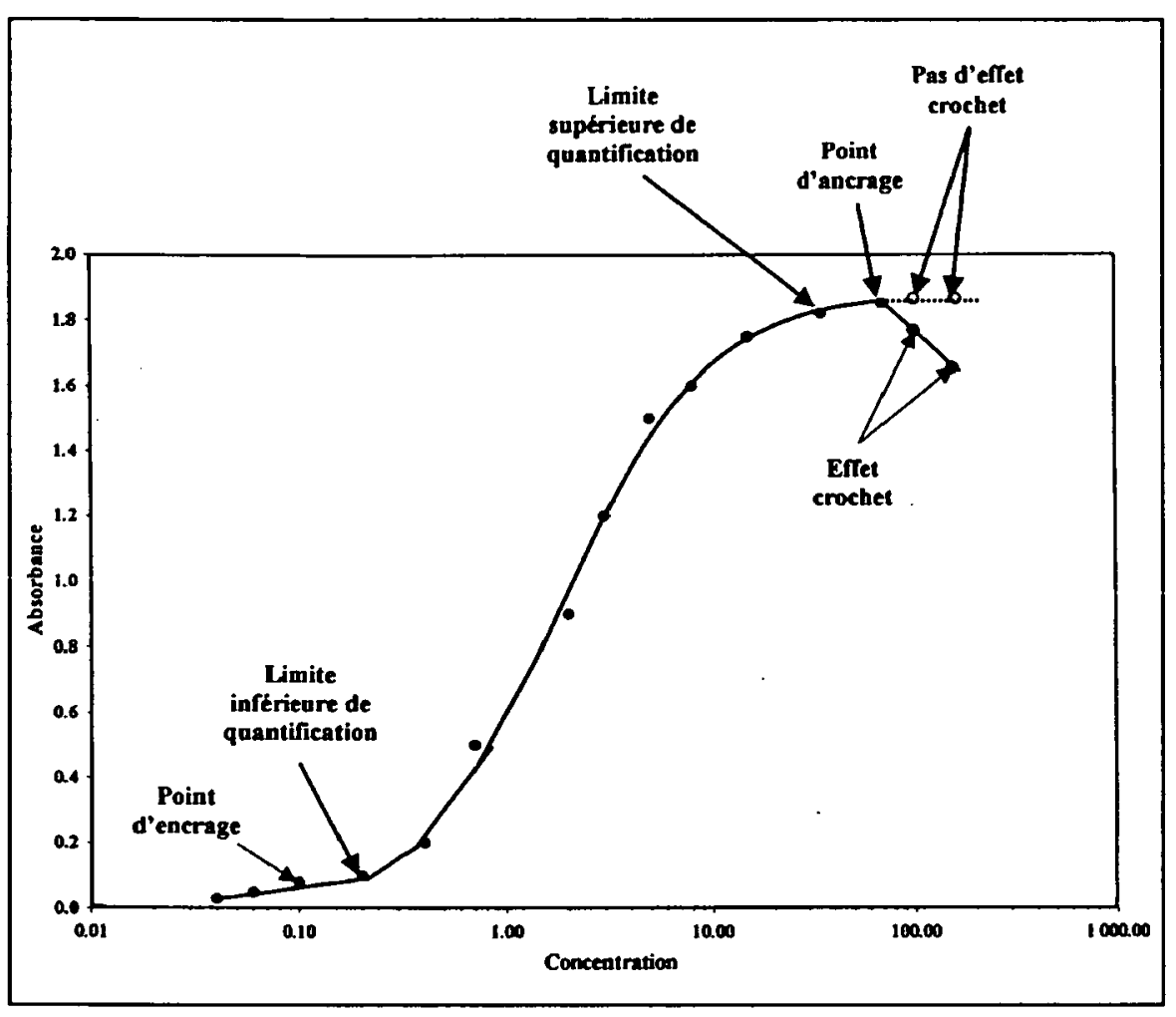

Figure 2 : Courbe sigmoïde type concentration/réponse et démonstration de l'effet crochet.

Pour les techniques immunologiques, deux limites de quantification déterminent l'intervalle des concentrations que l'on peut doser (Figure 2). Ce sont les plus petite et plus haute concentrations que l'on peut doser avec une fidélité et une exactitude acceptables. Etant donné la nature de la technique immunologique, l'intervalle de quantification est souvent très étroit, c'est pourquoi il est souvent nécessaire de diluer les échantillons. L'effet de la dilution et la linéarité dilutionnelle doivent être évalués à partir d'échantillons contenant des concentrations 100 à 1000 fois plus élevées que celles de la limite supérieure de quantification. Les dilutions de ces échantillons doivent être réalisées de façon à obtenir 1) une concentration au dessus de la limite supérieure de quantification afin d'évaluer «l'effet crochet» (diminution du signal causé par une forte concentration de l'analyte) (Figure 2) et 2) des concentrations dans la partie basse, moyenne et haute de la courbe afin d'évaluer la linéarité dilutionnelle. Il est admis en pratique que chaque étape de dilution ne peut pas excéder une dilution au $100^{\text {eme }}$.

\section{Exactitude :}

Les critères pour qu'une méthode de dosage soit acceptable (1) sont les suivants : au moins $67 \%$ (4 sur 6) des échantillons contrôle-qualité doivent avoir une erreur relative inférieure à $15 \%$ par rapport à la valeur théorique, soit $1 / 3$ des échantillons peuvent dépasser ces $15 \%$ d'erreur.

Pour chaque méthode de dosage, l'exactitude des résultats est le point le plus important. Parfois, plusieurs dosages du même échantillon contrôle-qualité peuvent être réalisés pour améliorer cette exactitude. Dans ce cas cette procédure doit être utilisée pendant la validation et lors du dosage des échantillons de concentrations inconnues.

\section{Autres recommandations :}

Si un pré-traitement de l'échantillon biologique est réalisé avant le dosage alors qu'il ne l'est pas pour la gamme d'étalonnage, il est important de déterminer le coefficient d'extraction et de l'utiliser pour exprimer les résultats. Plusieurs approches pour déterminer l'efficacité et la reproductibilité de l'extraction existent : 1) utilisation de l'analyte radiomarqué à l'état de trace et 2) utilisation d'un étalon interne non reconnu par l'anticorps et qui peut être dosé par une autre technique.

\section{Conclusion}

Dans ce manuscrit, les différentes étapes de la validation d'une méthode de dosage en bioanalyse sont décrites. Ce guide redéfinit les objectifs et les critères de la validation tout en permettant une approche pratique. Cette procédure de validation concerne essentiellement les méthodes chromatographiques (CG, CLHP) qui sont particulièrement bien adaptées au dosage des médicaments et de leurs métabolites dans les milieux biologiques. Quelques recommandations sont également fournies concernant les méthodes immunologiques. Les problèmes concernant la qualification de l'appareillage et le pré-traitement des échantillons sont abordés. Disposer d'une méthode de dosage correctement validée est un pré-requis, non seulement avant toute étude de pharmacocinétique ou de toxicocinétique et tout au long du développement d'un médicament mais aussi lors de l'utilisation de ce médicament en clinique. 


\section{Références}

1. http://www.fda.gov/cder/guidance/4252fnl.htm Guidance for industry. Bioanalytical method validation. May 2001. (consulté en avril 2004).

2. Shah V.P., Midha K.K., Findlay J.W.A., Hill H.M., Hulse J.D., McGilveray I.J., McKay G., Miller K.J., Patnaik R., Powell M.L., Tonelli A., Viswanathan C.T., Yacobi A. Bioanalytical method validation- A revisit with a decade of progress. Pharm. Res. $2000 ; 17$ : 1551-7.

3. Bressolle F., Bromet-Petit M., Audran M. Validation of liquid chromatographic and gas chromatographic method. Application to pharmacokinetic. J. Chromatogr. B Biomed. Appl. 1996 ; 686 : 3-10.

4. Causon R. Validation of chromatographic methods in biomedical analysis. Viewpoint and discussion. J. Chromatogr B. Biomed. Sci. Appl.. 1997 ; 689 : 175-80.

5. Shabir G.A. Validation of high-performance liquid chromatography methods for pharmaceutical analysis. Understanding the differences and similarities between validation requirements of the US food and drug administration, the US pharmacopeia and the international conference on harmonization. J. Chromatogr. A 2003 ; 987 : 57-66.

6. Hubert P., Nguyen-Huu J.J., Boulanger B., Chapuzet E., Chiap P., Cohen N., Compagnon P.A., Dewe W., Feinberg M., Lallier M., Laurentie M., Mercier N., Muzart G., Nivet C., Valat L. Validation des procédures analytiques quantitatives. Harmonisation des démarches. STP Pharma Pratiques 2003 ; 13 : 101-38.

7. Coursimault A., Blanchin M.D., Chevaleyre C., Coupeaud I., Delaire M., Dufat H., Hanras C., Icare M., Janicot J.L., Le Dantec E., Monier C., Portefaix D., Puig N. Qualification et maintenance d'une chaîne CLHP. Rapport d'une commission mixte ASFILAB/SFSTP. STP Pharma Pratiques 1998; 8:478-88.

8. International standard ISO/IEC 17025: general requirement for the competence of testing and calibration laboratories, International organization for standardization, 2000.

9. http://www.fda.gov/cder/guidance/5667fnl.htm. Guidance for Industry: part 11 ; electronic records, electronic signatures - scope and application, US Food and Drug Administration. August 2003. (consulté en avril 2004).

10.7 Paxton J.W. Protein binding of methotrexate in sera from normal human beings: effect of drug concentration, $\mathrm{pH}$, temperature, and storage. J. Pharmacol. Methods. $1981 ; 5: 203-13$.

11. Yeung C.Y., Lee F.T., Wong H.N. Effect of serum $\mathrm{pH}$ on bilirubin-protein binding. Acta Paediatr. Jpn. 1992; 34 : 23-7.

12. Brors O., Jacobsen S. pH lability in serum during equilibrium dialysis. Br. J. Clin. Pharmacol. 1985 ; 20 : 85-88.

13. Kearney A.S., Crawford L.F., Mehta S.C., Radebaugh G.W. The interconversion kinetics, equilibrium, and solubilities of the lactone and hydroxyacid forms of the HMG-CoA reductase inhibitor, CI-981. Pharm. Res. $1993 ; 10: 1461-5$.
14. Iglesias R., Villarroya F., Alemany M. Comparison of effects of different anticoagulants and sample handling procedures on rat insulin radioimmunoassay. Comp. Biochem. Physiol. A. 1985 ; 82 : 863-6.

15. Lagorce P., Perez Y., Ortiz J., Necciari J., Bressolle F. Assay method for the carboxylic acid metabolite of clopidogrel in human plasma by gas chromatography-mass spectrometry. J. Chromatogr. B Biomed. Sci. Appl. 1998 ; 720 : 107-17.

16. Buhrman D.L., Price P., Rudewicz P.J. Quantitatiion of CR27417 in human plasma using electrospray liquid chromatography tandem mass spectrometry: a study of ion suppression. J. Am. Soc. Mass Spectrom. 1996 ; 7 : 1099-105.

17. King R., Bonfiglio R., Fernandez-Metzler C., MillerStein C., Olah T. Mechanistic investigation of ionization suppression in electrospray ionization. J. Am. Soc. Mass Spectrom. $2000 ; 11: 942-50$.

18. Dams R., Huestis M.A., Lambert W.E., Murphy C.M. Matrix effect in bio-analysis of illicit drugs with LCMS/MS: influence of ionization type, sample preparation, and biofluid. J. Am. Soc. Mass Spectrom. 2003 ; 14 : 1290-4.

19. Jemal M., Schuster A., Whigan D.B. Liquid chromatography/tandem mass spectrometry methods for quantitation of mevalonic acid in human plasma and urine: method validation, demonstration of using a surrogate analyte, and demonstration of unacceptable matrix effect in spite of use of a stable isotope analog internal standard. Rapid Commun. Mass Spectrom. 2003 ; 17 : 1723-34.

20. Bressolle F., Pistre M.C., Brès J., Sabatier R. Quantitative thin-layer and high performance liquid chromatographic determination of lorapride in biological fluids and a pharmacokinetic study in humans. J. Pharm. Sci. 1985 ; 74 : 1215-8.

21. Hamel B., Audran M., Costa P., Bressolle F. Reversedphase high-performance liquid chromatographic determination of enoxacin and 4-oxo enoxacin in human plasma and prostatic tissue. Application to a pharmacokinetic study. J. Chromatogr.A $1998 ; 812$ : 369-75.

22. Nijhuis A., van der Knap H.C.M., de Jong S., Vandeginst B.G.M. Strategy for ruggedness tests in chromatographic method validation. Analytica Chimica Acta 1999 ; 391 : 187-202.

23. Vander Heyden Y., Questier F., Massart L. Ruggedness testing of chromatographic methods : selection of factors and levels. J. Pharm. Biomed. Anal. 1998 ; $18: 43-56$

24. Desilva B., Smith W., Weiner R., Kelley M., Smolec J.M., Lee B., Khan M., Tacey R., Hill H., Celniker A. Recommendations for the bioanalytical method validation of ligand-binding assays to support pharmacokinetic assessments of macromolecules. Pharm. Res. $2003 ; 20$ : 1885-900.

25. Findlay J.W.A., Smith W.C., Lee J.W., Nordblom G.D., Das I., Desilva B.S., Khan M.N., Bowsher R.R. Validation immunoassays for bioanalysis : a pharmaceutical industry perspective. J. Pharm. Biomed. Anal. $2000 ; 21: 1249-73$. 